\title{
Análise de Risco em Comercialização de Energia Elétrica
}

\section{Lyz Leite ${ }^{\mathbf{1 , 2}}$ https://orcid.org/0000-0002-7623-1249}

\section{Eduardo Sodré ${ }^{1,3}$ https://orcid.org/0000-0002-5793-8900}

\author{
${ }^{1}$ Escola Politécnica de Pernambuco, Universidade de Pernambuco, Recife, Brasil. \\ ${ }^{2}$ Kroma Energia, Recife, Brasil. \\ ${ }^{3}$ Companhia Hidroelétrica do São Francisco, Recife, Brasil. \\ E-mail do autor principal: Lyz Leite lyz.leite@outlook.com.br
}

\section{RESUMO}

Este artigo tem como objetivo uma introdução à área de análise de risco numa empresa de comercialização de energia elétrica. A análise aqui apresentada segue na linha dos trabalhos de análise de risco para o mercado financeiro, sendo esta a metodologia de análise de risco que mais se adequa à realidade de uma empresa de comercialização de energia elétrica. Os pontos levantados neste trabalho são o de Análise Risco x Retorno e métricas para tomada de decisão sob incerteza.

PALAVRAS-CHAVE: Análise de Risco; Comercialização; Tomada de Decisão sob Incerteza;

\section{ABSTRACT}

This article aims at an introduction to the area of risk analysis in an electric energy trading company (retailing). The analysis presented here follows the line of risk analysis for the financial market. This being the methodology of risk analysis that best suits the real life an electric energy trading company. The points raised in this work are the risk $x$ return analysis and metrics for decision making under uncertainty.

KEY-WORDS: Risk Analysis; Electricity Trading; Decision Making under Uncertainty; 


\section{Introdução}

Os contratos de compra e venda de energia elétrica firmados entre os agentes levam em consideração, entre outros fatores, a expectativa que cada agente tem quanto ao Preço de Liquidação das Diferenças - PLD durante a vigência de cada contrato.

O PLD, por sua vez, é uma derivada de quanto custa a operação do sistema naquele momento e reflete, de certa forma, o que está previsto de despacho termoelétrico e hidrelétrico para a próxima semana operativa. Sua alta volatilidade decorre da dependência hidrológica em seu cálculo [1].

$\mathrm{Na}$ análise de um portfólio de comercialização de energia devem ser levados em consideração a volatilidade dos preços do PLD para o período de vigência dos contratos e riscos associados a atividade, bem como a liquidez, o risco de mercado, o risco da contraparte, o risco político/regulatório, entre outros [2].

Esse artigo abordará nas seções seguintes como se dá a formação do PLD e quais são as métricas de risco mais usuais no mercado de energia elétrica no Brasil.

\section{FORMAÇÃO DO PREÇO DE LIQUIDAÇÃO DAS DIFERENÇAS}

O Preço de Liquidação das Diferenças - PLD é utilizado para a valoração dos montantes liquidados no Mercado de Curto Prazo - MCP. O PLD é apurado pela CCEE, por submercado, conforme determina o Decreto 5.163/2004. A base para cálculo do PLD é o Custo Marginal de Operação - CMO, fruto dos modelos matemáticos utilizados pelo Operador Nacional do Sistema Elétrico - ONS para definir a programação da operação do sistema, limitado a um preço mínimo e por um preço máximo, estabelecidos anualmente pela Agência Nacional de Energia Elétrica - ANEEL.

\subsection{Planejamento Anual Energético}

O PEN é realizado com periodicidade anual e revisões quadrimestrais. Com base em informações, recebidas das autoridades setoriais e dos agentes associados, sobre a oferta futura de energia e sobre as previsões de consumo do mercado, o ONS utiliza modelos matemáticos de otimização e simulação da operação paradeterminar estratégias operativas e avaliação das condições de suprimento ao SIN.
Nesse estudo são realizadas avaliações probabilísticas do atendimento ao mercado, em que se calculam, inclusive, os riscos de ocorrência de déficits.

Os dados e informações utilizados nos estudos para o PEN também são empregados no processamento do modelo de médio prazo, no âmbito da elaboração do Programa Mensal de Operação - PMO.

\subsection{Planejamento Mensal da Operação}

O PMO é elaborado pelo ONS com a participação dos agentes envolvidos, em reunião conjunta, registrada em ata, para garantir a transparência do processo. Os estudos realizados em base mensal e revistos semanalmente - fornecem metas e diretrizes eletro energéticas de curto prazo, de modo a otimizar a utilização dos recursos de geração e transmissão do SIN, segundo procedimentos e critérios consubstanciados nos Procedimentos de Rede, homologados pela ANEEL.

\subsection{Modelo NEWAVE}

O NEWAVE é um modelo de planejamento da operação de sistemas hidrotérmicos com representação individualizada do parque termelétrico e representação agregada, por meio de reservatórios equivalentes de energia, do parque hidrelétrico. As usinas hidrelétricas de cada submercado são representadas por meio de um único reservatório equivalente. As linhas de transmissão que conectamos submercados são representadas por meio de limites de intercâmbio.

O objetivo básico do NEWAVE é determinar metas de geração que atendam a demanda e minimizem o valor esperado do custo de operação ao longo do período de planejamento.

Um dos principais resultados obtidos na estratégia de solução do modelo NEWAVE é a função de custo futuro. É por meio dessa função que 0 encadeamento com o modelo de curto prazo - DECOMP - é realizado, compatibilizando a política de operação de curto prazo com a política de operação de médio prazo, definida pelo NEWAVE. 


\subsection{Modelo DECOMP}

O objetivo do DECOMP é determinar o despacho de geração das usinas hidráulicas e térmicas que minimiza o custo de operação ao longo do período de planejamento, dado o conjunto de informações disponíveis (carga, vazões, disponibilidades, limites de transmissão entre subsistemas, função de custo futuro do NEWAVE).

A Figura 1 ilustra graficamente a cadeia dos modelos computacionais utilizados no Brasil para a formação dos Preços de Liquidação de Diferenças (PLD) do Mercado de Curto Prazo (Mercado SPOT).

Vale ressaltar que o Modelo DESSEM ainda está em discussão e testes no Setor Elétrico Brasileiro e sua utilização está prevista para o ano de 2020. Onde então o preço da energia elétrica no Brasil passará a ser um preço semi-horário, conforme a maioria dos mercados de energia elétrica mais desenvolvidos ao redor do mundo.



Figura 1: Cadeia de Modelos Computacionais para determinação do PLD.

Fonte: (ONS).

\section{CURVA FORWARD DE PREÇOS DE ENERGIA ELÉTRICA}

A curva Forward é um preço de referência de energia para determinado produto, em cada momento no tempo, que serve como base para precificação de operações ou valoração de ativos e está relacionado com o conceito de "marcação ao mercado".

Matematicamente, uma curva Forward é uma função que depende de duas variáveis: o período de apuração e a maturidade ou distância até o suprimento. Por exemplo, pode-se ver na Figura 2 , que a energia convencional que está sendo negociada hoje, para entrega em novembro de 2019 no Submercado SE/CO (Sudeste/Centro Oeste) está custando 216,13 R\$/MWh.
Esse preço é definido a partir da interação da oferta e demanda de energia no mercado em diferentes horizontes e varia de acordo com a conjuntura do mercado.

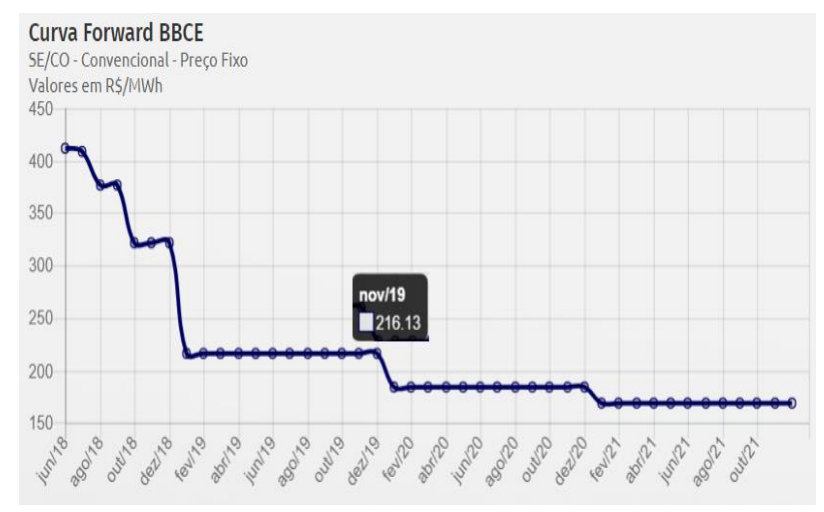

Figura 2: Curva Forward. Fonte: (BBCE).

\section{ANÁLISE DE RISCO}

A gestão de risco se mostra uma atividade complexa e importante, envolvendo fatores delimitados pelas regras de cada mercado, e também fatores como a existência de instrumentos financeiros capazes de proporcionar hedge aos agentes, contando com inúmeras métricas de risco para subsidiar a tomada de decisão $[3,4]$.

A análise de Risco compõem-se basicamente da busca de 2 (duas) informações:a chance (probabilidade);e o impacto (valor monetário) de eventos desfavoráveis ou favoráveis ocorrerem. Toda vez que se está trabalhando com Risco é importante ter-se em mente estas duas dimensões. Análise de Risco é uma Tomada de Decisão sob Incerteza.

Essa seção abordará os principais tipos de análise de risco do mercado de energia herdadas do mercado financeiro. Estas análises são utilizadas para controlar ou minimizar o risco na tomada de decisão.

Sabe-se que a comercialização de energia está submetida a diversos cenários de incerteza. Estes cenários podem ser quantificados em termos de probabilidade de ocorrência. Seguindo na linha da análise tradicional do Mercado Financeiro, podemos exemplificar através da Figura 3 a Taxonomia da Análise de Risco. 


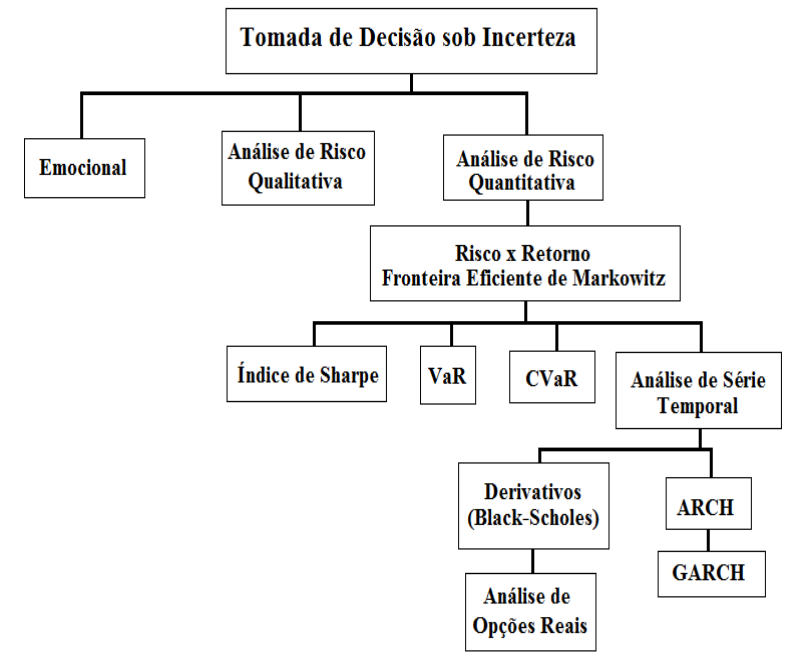

Figura 3: Taxonomia da Análise de Risco Financeira, Fonte: Os Autores.

Entre as incertezas associadas a um portfólio de comercialização de energia, temos como exemplo: demanda; PLD; Curva Forward; tipo do contrato (Fixo, Prêmio, Swap); etc. Apesar da quantificação do risco de uma carteira de comercialização ser uma tarefa que necessita de um pouco de arte (bom-senso) tanto quanto de ciência, temos certeza absoluta que a Análise de Risco Quantitativo é imprescindível para a segurança da empresa de comercialização e para o estabelecimento de estratégias adequadas para a tomada de decisão.

Neste trabalho vamos exemplificar um caso de Análise de Risco que está em linha com a Análise de Série Temporal e o cálculo do "Value at Risk" $(\mathrm{VaR})$.

Nas seções seguintes vamos explicar de forma simplifica algumas métricas de risco que auxiliam na Tomada de Decisão em uma Comercializadora de Energia Elétrica.

\subsection{Downside Risk e Semivariance}

Em problemas de comercialização a preocupação é com os desvios negativos com relação ao esperado, pois, estes que causam as perdas financeiras. Assim, essa medida é utilizada para visualizarmos a média dos cenários abaixo de um determinado ponto de corte.

A variância de uma distribuição de retorno mede o desvio dos retornos reais do retorno esperado. Esta é a tradicional Análise Risco (desvio-padrão) x Retorno (valor esperado dos retornos) criada por Markowitz [5].

Ao estimar a variância, consideramos não apenas os retornos que caem abaixo do retorno médio (downside risk), mas também os que estão acima dele (upside risk). As comercializadoras geralmente consideram como risco os retornos que estão abaixo do retorno médio, ou seja, o downside risk.

Existe uma outra métrica conhecida como Semivariance, que considera somente 0 downside risk. Para estimar a semivariância, são calculados somente os desvios dos retornos em relação ao retorno médio, somente se o retorno for menor que o retorno médio. Não são considerados os retornos que são maiores que 0 retorno médio. Para maiores detalhes, e para a fórmula de cálculo da Semivariance procurar na referência [6].

\subsection{Valor em Risco ( VaR) e Valor em Risco Condicional(CVaR)}

\author{
Conforme Damodaran [7]:
}

\begin{abstract}
Embora o termo "Value at Risk" não tenha sido amplamente utilizado antes de meados da década de 1990, as origens da medida recaem num tempo bastante remoto. As matemáticas subjacentes ao VaR foram amplamente desenvolvidas no contexto da teoria de portfólios por Harry Markowitz e outros, embora seus esforços tenham sido direcionados para um fim diferente - criar portfólios ótimos para investidores em ações. Em particular, o foco nos riscos de mercado e os efeitos das operações nesses riscos são centrais para como o cálculo do VaR.
\end{abstract}

O cálculo do VaR (Value at Risk) é realizado de uma forma bem simples. Dada a distribuição de Probabilidades dos Ganhos/Perdas do negócio em análise, podemos calcular o valor da abscissa que determina, por exemplo, um valor de área à direita, na curva de distribuição de probabilidades, em 95\%. Essa valor de abscissa seria o VaR95\%. Em outras palavras, o valor de abscissa $\mathrm{VaR}_{95 \%}$ determina que a probabilidade de ocorrer retornos maiores ou igual ao $\mathrm{VaR}_{95} \%$ é de $95 \%$ de chance (Figura 4 ).

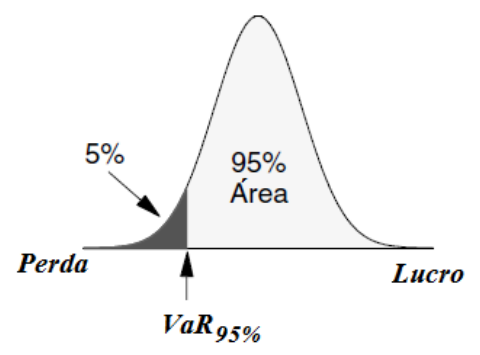

Figura 4: VaR.

Fonte: Os Autores.

O CVaR95\% (Conditional Value at Risk) é o valor esperado de todas as distribuições de 
probabilidade que estão abaixo do $\mathrm{VaR}_{95 \%}$. Para cálculo do $\mathrm{CVaR}_{95 \%}$ pode ser utilizada a seguinte fórmula:

$$
\operatorname{CVaR}_{95 \%}=\sum_{i \in \Omega} P i . X i
$$

onde:

$\Omega$ : é o conjunto de todos os valores da Distribuição de Probabilidade que estão abaixo do $\mathrm{VaR}_{95 \%}$;

$X_{i}$ : são os valores de retorno (valores da abscissa) abaixo do $\mathrm{VaR}_{95 \%}$;

$P_{i}$ : são os valores de probabilidade para os $X_{i}$ 's

Vê-se que o $C V a R_{95 \%}$ não é um valor de abscissa, assim como é o $V a R_{95 \%}$. O objetivo principal do $C V a R$ é "quantificar" o valor médio das perdas ao qual a empresa está submetida. A Figura 5 ilustra bastante bem o conceito do CVaR. Vê-se na Figura 5 que as duas distribuições de probabilidades tem o mesmo $V a R_{\alpha \%}$, mas possuem $C V a R$ diferentes. Isso significa que para alguns casos particulares, o CVaR é uma métrica de risco que deve ser usada no lugar da métrica VaR.

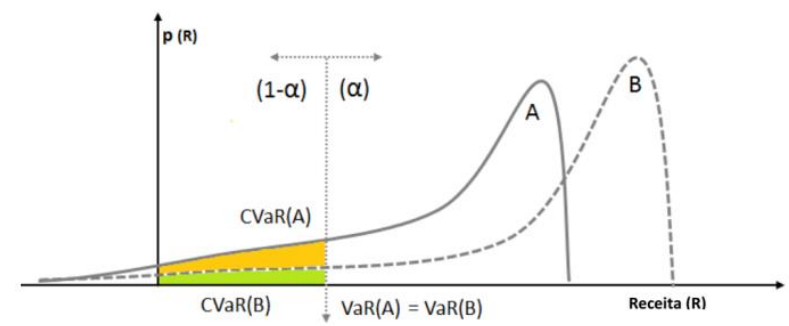

Figura 5: Var e CVaR.

Fonte: (CCEE).

\section{RESULTADOS}

As operações no mercado livre de energia brasileiro acontecem baseadas na expectativa que os agentes têm quanto ao valor do Preço de Liquidação das Diferenças - PLD, que é o preço de curto prazo da eletricidade.

O PLD apresenta alta volatilidade devido à sua dependência de características físicas singulares, tais como:

- Casamento em tempo real de demanda e produção;

- A incerteza da demanda;

- A inelasticidade de preço da demanda;

- A hidrologia;

- O longo tempo de resposta (lead-time) necessário para a expansão da geração a fim de atender ao crescimento da demanda.

Essas características próprias do mercado de eletricidade fazem com que a probabilidade de ocorrência de picos súbitos e temporários de preço no mercado à vista seja maior que em quaisquer outros mercados de commodities e causam uma extraordinária alta volatilidade [8].

Essa particularidade do mercado de energia faz com que ferramentas muito utilizadas em outros mercados para auxílio da gestão de risco sejam pouco perspicazes quando utilizadas para uma visão genérica de portfólios de energia e necessitem ser adaptadas para o Mercado Brasileiro de Energia Elétrica.

Por exemplo, a abordagem de Monte Carlo para simular portfólio sorteando o PLD em função de sua probabilidade associada, tem que ter os cálculos das probabilidades do PLD analisadas com bastante cuidado e atenção. É certo afirmar que o cálculo das distribuições de probabilidade do PLD, num horizonte de longo-prazo, deve levar em conta vários cenários criados e balizados pela experiência dos especialistas, e não somente cenários vindos diretamente de um $D E C K$ do NEWAVE do PMO.

Também podemos afirmar que as Técnicas de Análise de Série Temporal são mais adequadas para uma Análise de Risco de mais curto prazo.

O que é desejável para tomada de decisões de uma empresa é a responsabilidade. Deve ser ideal que a aversão ao risco seja definida em conselho. E que esse processo possa ter bastante transparência, e que possa ser auditável e reprodutível. Também é importante lembrar que a Tomada de Decisão deve ser testada e simulada ex-ante a fim de minimizar o fator emocional.De modo que, o modelo a ser desenvolvido deve levar em consideração o perfil de risco da empresa e consiga reproduzir as decisões subjetivas quantitativamente.

Para o exemplo apresentado abaixo, temos uma Comercializadora em posição vendida de $5 \mathrm{MWmed}$ de energia para 0 trimestre julho/agosto/setembro de 2018, conforme Tabela 1.

Tabela 1: Posição Vendida da Comercializadora

\begin{tabular}{|l|r|}
\hline Data & $12-$ Jun \\
\hline Preço (R\$/MWh) & 375.00 \\
\hline Resultado Operacional (R\$) & $600,000.00$ \\
\hline Exposição (MWmed) & -5.00 \\
\hline Horas de julho, agosto e setembro & 2208.00 \\
\hline
\end{tabular}

Na Tabela 2 estão apresentados os valores da Série Temporal de preços extraída da BBCE. Essa 
é a série que será utilizada para a Análise de Risco.

Tabela 2: Série Temporal do Preço da Energia para o 3TRI (julho/agosto/setembro).

\begin{tabular}{|c|c|c|c|c|c|}
\hline Data & Fechamento & Retorno & Data & Fechamento & Retorno \\
\hline 26-Feb & 244.00 & $-1.67 \%$ & 4-May & 230.00 & $-2.68 \%$ \\
\hline 27-Feb & 240.00 & $7.34 \%$ & 7-May & 224.00 & $-2.05 \%$ \\
\hline 5-Mar & 259.00 & $0.77 \%$ & 8-May & 219.50 & $-0.69 \%$ \\
\hline 12-Mar & 261.00 & $-1.56 \%$ & 9-May & 218.00 & $1.36 \%$ \\
\hline 13-Mar & 257.00 & $-3.21 \%$ & 10-May & 221.00 & $9.05 \%$ \\
\hline 22-Mar & 249.00 & $-2.47 \%$ & 14-May & 243.00 & $7.25 \%$ \\
\hline 26-Mar & 243.00 & $-1.67 \%$ & 15-May & 262.00 & $2.60 \%$ \\
\hline 27-Mar & 239.00 & $-11.16 \%$ & 16-May & 269.00 & $3.58 \%$ \\
\hline 28-Mar & 215.00 & $0.92 \%$ & 17-May & 279.00 & $6.38 \%$ \\
\hline 29-Mar & 217.00 & $-1.88 \%$ & 18-May & 298.00 & $0.67 \%$ \\
\hline 2-Apr & 213.00 & $0.93 \%$ & 21-May & 300.00 & $0.50 \%$ \\
\hline 3-Apr & & $2.27 \%$ & 22-May & & $-0.50 \%$ \\
\hline 5-Apr & 220.00 & $0.90 \%$ & 23-May & 300.00 & $-0.67 \%$ \\
\hline 6-Apr & 222.00 & $5.53 \%$ & 24-May & 298.00 & $1.97 \%$ \\
\hline 9-Apr & 235.00 & $-13.53 \%$ & 25-May & 304.00 & $-5.56 \%$ \\
\hline 12-Apr & 207.00 & $5.69 \%$ & 28-May & 288.00 & $4.32 \%$ \\
\hline 17-Apr & 219.50 & $-0.69 \%$ & 30-May & 301.00 & $2.59 \%$ \\
\hline 18-Apr & 218.00 & $0.91 \%$ & 1-Jun & 309.00 & $4.89 \%$ \\
\hline 19-Apr & 220.00 & $0.00 \%$ & 4-Jun & 324.90 & $1.55 \%$ \\
\hline 20-Apr & 220.00 & $0.00 \%$ & 5-Jun & 330.00 & $0.90 \%$ \\
\hline 24-Apr & 220.00 & $-1.38 \%$ & 6-Jun & 333.00 & $1.04 \%$ \\
\hline 25-Apr & 217.00 & $0.46 \%$ & 7-Jun & 336.50 & $0.74 \%$ \\
\hline 27-Apr & 218.00 & $11.02 \%$ & 8-Jun & 339.00 & $6.35 \%$ \\
\hline 3-May & 245.00 & $-6.52 \%$ & 11-Jun & 362.00 & $0.00 \%$ \\
\hline
\end{tabular}

Dada a distribuição de probabilidades do Retorno do valor da energia conforme a curva de preços negociada no Mercado, conforme Figura 6, podemos calcular a Distribuição de Probabilidades dos Ganhos/Perdas para essa posição vendida da Comercializadora, conforme Figura 7.

O exemplo ilustra que para o curto prazo, a volatilidade do mercado indica um viés de alta de preços, aconselhando quantitativamente a tomada de decisão no dia da análise, ou seja, a comercializadora deveria comprar imediatamente a energia para sanar sua exposição.

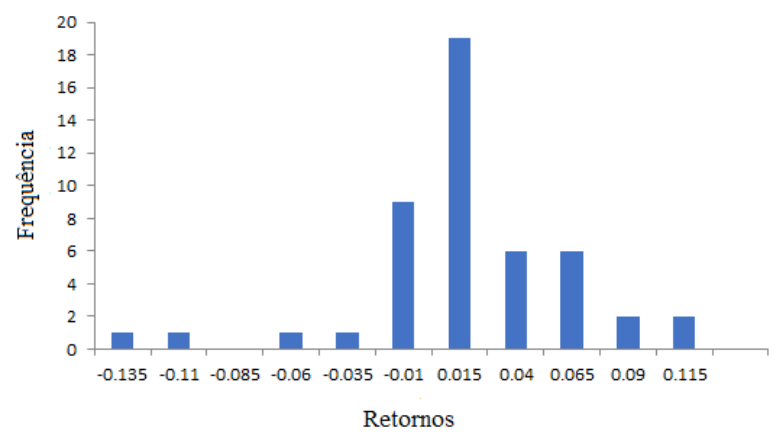

Figura 6: Distribuição de Probabilidades dos Retornos, Fonte: Os Autores.

Também devemos ressaltar, mais uma vez, que a Tomada de Decisão é muito mais do que uma simples análise do Valor Esperado. A Tomada de Decisão sob Incerteza de uma
Comercializadoratambém engloba outros fatores que não são facilmente avaliados diretamente. $O$ mercado de energia oscila por fatores externos à expectativa do PLD, tais como, mudança regulatória e/ou grande oferta, leilões de venda ou MCSD, por exemplo. No entanto, o fundamento para a formação do PLD predomina e é o maior balizador para a decisão.

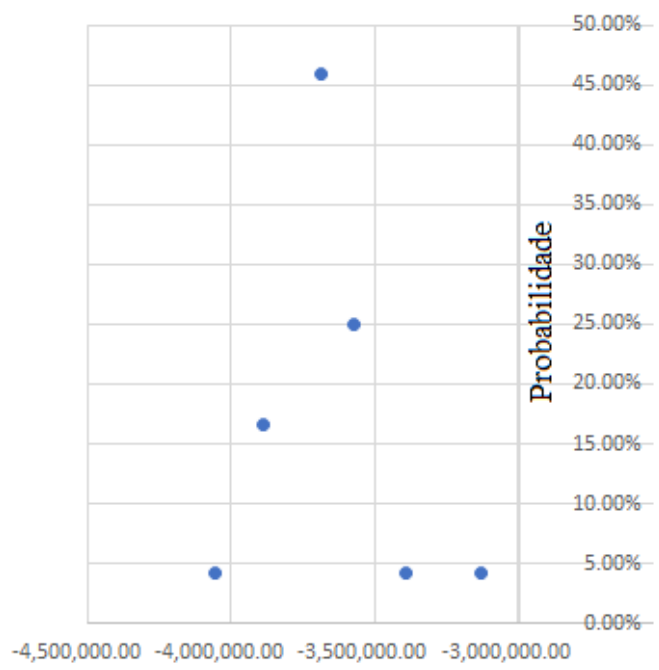

Valores em $\mathrm{R} \$$ dos Ganhos/Perdas

Figura 7: Distribuição de Probabilidades dos Ganhos/Perdas.

Fonte: Os Autores.

A gestão de risco deve ser modelada à realidade de cada empresa, pois, o resultado individual de cada métrica é ponderado conforme a aversão ao risco destas. Enquanto algumas empresas consideram de extrema importância a manutenção de caixa operacional mínimo outras acreditam que a forma correta de operar é seguindo um rígido "stoploss".

Como dito, a hidrologia é o fator determinante na formação do PLD. Os mapas hidrológicos que preveem mais ou menos precipitação tendenciam os preços para baixo ou para cima, respectivamente. A visão de confiança que a meteorologia fornece mostra dez dias a frente, um estudo de outros fatores hidrológicos, tais como, pressão, umidade e solo estende esse horizonte, sendo uma opção segura, esperar a semana seguinte para realizar a compra necessária se esta der indícios de boa precipitação.

\section{CONCLUSÃO}

O mercado de energia elétrica no Brasil é relativamente novo, mesmo com seu marco em 1995 ascendeu somente após a crise de 
abastecimento em 2002, e hoje encontra-se um ambiente de contratação muito competitivo.

Os agentes que compõe o Mercado Livre sejam eles geradores, consumidores e/ou comercializadores - operavam em simultâneo com a criação de regras que, em seguida, iriam reger - mercado. A própria dificuldade em regulamentação eficiente motivou diversas mudanças que continuam ocorrendo no setor.

Os riscos do mercado de energia, além do famigerado PLD, englobam: liquidez; regulamentação; crédito; tributação; operacional; entre outros, inerentes ao mercado e pouco mitigáveis.

É importante ressaltar que a Análise de Risco (conforme as técnicas herdadas do Mercado Financeiro) não é uma resposta única e definitiva para a operação de uma Comercializadora de Energia. As Técnicas de Análise de Risco são um subsídio, entre tantos outros, para ajudar na Tomada de Decisão. As métricas de risco ( $V a R$, $C V a R$, etc.) não são a Decisão.

Dito isto, podemos declarar que estamos convencidos da importância da utilização de Técnicas de Análise de Risco para subsidiar a Tomada de Decisão de curto, médio e longo prazo de uma Comercializadora de Energia Elétrica. E o mais importante, é que a Análise de Risco traz para a mesa de discussão uma nova forma de olhar e entender o negócio da empresa.

\section{REFERÊNCIAS}

[1] TOLMASQUIM, M. T. Novo Modelo do Setor Elétrico Brasileiro. 2. ed. Synergia Editora, 2015.

[2] KOVACEVIC, R M.; PFLUG, G. C.; VESPUCCI, M. T. (Ed.). Handbook of Risk Management in Energy Production and Trading. Springer, 2013.

[3] GUDER, R. Otimização de Portfólios de Contratos De Energia Elétrica Utilizando Algoritmos Genéticos Multiobjetivo. 2009. Dissertação (Mestrado em Engenharia Elétrica) - Universidade Federal de Santa Catarina, Florianópolis, 2009.

[4] TORRES, R. C. Avaliação de Portfolios de Contratos de Compra e Venda de Energia Elétrica: Uma Abordagem pela Teoria de Opções. Dissertação (Mestrado em Engenharia Industrial) - Pontifica Universidade Católica, Rio de Janeiro, 2006.

[5] MARKOWITZ, H. Portfolio Selection. The Journal of Finance, v. 7, 1952.
[6] DAMODARAN, A. Applied Corporate Finance. 4. ed. Hoboken, NJ: Wiley, Nob. 2014.

[7] DAMODARAN, A. Strategic Risk Taking: A Framework for Risk Management. New Jersey: FT Press, august 2007.

[8] MAYO, R. Derivativos de Eletricidade \& Gerenciamento de Risco, Synergia, 2009. 